xiv

\title{
THE ORGANIZING COMMITTEE
}

\section{SCIENTIFIC}

George Djorgovski (USA)

Ken Freeman (Australia)

Hyung-Mok Lee (Korea)

Douglas C. Heggie (UK)

Piet Hut (USA)

Shogo Inagaki (Japan)

Steve McMillan (USA)

Georges Meylan (Germany)

Harvey B. Richer (Canada)

Daiichiro Sugimoto (Japan, Chairperson)

Frank Verbunt (Netherlands)

\section{LOCAL}

Izumi Hachisu (University of Tokyo)

Junichiro Makino (University of Tokyo, chairperson)

Makoto Taiji (University of Tokyo)

The symposium was sponsored by IAU Commission 37 and co-sponsored by IAU Commissions 28, 30 and 35 . 